PROCEEDINGS OF THE

AMERICAN MATHEMATICAL SOCIETY

Volume 137, Number 9, September 2009, Pages 2817-2823

S 0002-9939(09)09780-9

Article electronically published on April 10, 2009

\title{
RESTRICTED LIE ALGEBRAS IN WHICH EVERY RESTRICTED SUBALGEBRA IS AN IDEAL
}

\author{
SALVATORE SICILIANO
}

(Communicated by Gail R. Letzter)

\begin{abstract}
We characterize restricted Lie algebras over perfect fields all of whose restricted subalgebras are ideals.
\end{abstract}

\section{INTRODUCTION}

The study of the influence of given systems of subgroups of a group on the structure of the group itself is one of the most important topics in group theory. Motivated by such results, Lie algebras whose subalgebras are subject to specific conditions have been extensively studied over the decades (see e.g.: 1, 2, 5, 6, 7, 9, 17, 18, 20, 21, 22, 23). Furthermore, more recently a number of problems from group theory have been considered in the category of restricted Lie algebras as well (see e.g. 4, 10, 13, 14, 15, 16]).

We recall that the structure of groups in which every subgroup is normal has been completely described by R. Dedekind and R. Baer (see [8] and [3]). In the same framework, the structure of associative, alternative and Jordan algebras in which every subalgebra is an ideal was discussed by S.-H. Liu in 11, and the same question for power-associative algebras was considered by D.L. Outcalt in [12].

Now, it is immediate to see that a Lie algebra in which all subalgebras are ideals is necessarily abelian. On the other hand, the restricted version of this problem turns out to be more interesting.

In this paper we investigate the class $\mathcal{D}$ of restricted Lie algebras with the property that every restricted subalgebra is a (restricted) ideal. In particular, a characterization of such restricted Lie algebras over perfect fields is obtained. For a restricted Lie algebra $(L,[p])$ over a field of characteristic $p>0$, we denote by $Z(L)$ the center of $L$, by $L^{\prime}$ the derived subalgebra of $L$, and by $\mathcal{N}(L)$ the null cone of $L$, namely, the set of all zeros of $[p]$. For a subset $S$ of $L$, we denote by $S_{p}$ the restricted subalgebra generated by $S$ and write $L^{[p]}:=\left\langle x^{[p]} \mid x \in L\right\rangle_{p}$. A restricted subalgebra $H$ of $L$ is called cyclic if $H=\langle x\rangle_{p}$ for some $x \in H$. Our main result is the following:

Theorem 1.1. Let $(L,[p])$ be a restricted Lie algebra over a perfect field $F$ of characteristic $p>0$. Then every restricted subalgebra of $L$ is an ideal if and only if one of the following conditions is satisfied:

Received by the editors May 19, 2008.

2000 Mathematics Subject Classification. Primary 17B05, 17B50.

Key words and phrases. Restricted subalgebra, restricted ideal, 2-closed field.

(C)2009 American Mathematical Society Reverts to public domain 28 years from publication 
(i) $L$ is abelian;

(ii) $p=2, L$ is nilpotent of class 2 and $L=T \oplus H$, where $T$ is a torus and $H$ a 2-nil restricted Lie algebra such that $H_{2}^{\prime}$ is cyclic, $H_{2}^{\prime}=H^{[2]}$, and $\mathcal{N}(H) \subseteq Z(H)$.

We shall also establish for which fields the class of restricted Lie algebras satisfying condition (ii) of Theorem 1.1 is actually not empty. Finally, we shall show that Theorem 1.1 fails without the assumption of perfectness of the ground field.

\section{PRoOF OF THE THEOREM AND CONCLUDING REMARKS}

Let $(L,[p])$ be a restricted Lie algebra over a field of characteristic $p>0$. An element $x$ of $L$ is said to be $p$-algebraic if $\operatorname{dim}\langle x\rangle_{p}<\infty$. If $x$ is $p$-nilpotent, the minimal $n$ such that $x^{[p]^{n}}=0$ is called the exponent of $x$.

It turns out that the restricted Lie algebras in $\mathcal{D}$ are nilpotent of class at most 2. Indeed, one has

Proposition 2.1. Let $(L,[p])$ be a non-abelian restricted Lie algebra over a field $F$ of characteristic $p>0$ such that every restricted subalgebra of $L$ is an ideal. Then $L^{\prime} \subseteq L^{[p]} \subseteq Z(L)$ and every element of $L$ is $p$-algebraic.

Proof. Let $H$ be a subalgebra of $L$. By [19] (Chapter 2, Proposition 1.3), $H$ is an ideal of the restricted subalgebra $H_{p}$ of $L$ and then, by the hypothesis, $H$ is a 2-step subideal of $L$. Thus, it follows from [18] that $L$ is nilpotent of class 2, and $L^{[p]}$ is a restricted ideal of $L$ contained in $Z(L)$. Put $\mathfrak{L}:=L / L^{[p]}$ and let $x, y \in \mathfrak{L}$. Then $\mathfrak{L} \in \mathcal{D}$ and therefore $F x$ and $F y$ are both ideals of $\mathfrak{L}$. It follows that $[x, y] \in F x \cap F y$; hence one necessarily has $[x, y]=0$. Thus $\mathfrak{L}$ is abelian, so that $L^{\prime} \subseteq L^{[p]}$, and the first part of the claim follows.

Now, let $a, b$ be non-commuting elements of $L$ and put $c:=[a, b]$. As $L \in \mathcal{D}$ we have $c \in\langle a\rangle_{p}$, so that

$$
c=\sum_{i=0}^{n} \alpha_{i} a^{[p]^{i}}
$$

for some $\alpha_{0}, \ldots, \alpha_{n} \in F$. Assume, if possible, that $a$ is not $p$-algebraic. As $L \in \mathcal{D}$, by the first part we have $c=\left[a+a^{[p]^{n+1}}, b\right] \in\left\langle a+a^{[p]^{n+1}}\right\rangle_{p}$. Since $\left\{a^{[p]^{i}} \mid i \geq 0\right\}$ is an $F$-linearly independent set, by (2.1) the previous conclusion is impossible; hence $a$ is a $p$-algebraic element of $L$.

Finally, suppose, if possible, that $Z(L)$ contains a non-p-algebraic element $u$. Then there exists a positive integer $m$ such that $\langle a\rangle_{p} \cap\left\{u^{[p]^{j}} \mid j \geq m\right\}=\{0\}$, in particular $\left[a+u^{[p]^{m}}, b\right]=c \notin\left\langle a+u^{[p]^{m}}\right\rangle_{p}$, contradicting the fact that $L$ is in $\mathcal{D}$.

Proof of Theorem 1.1. We first show sufficiency. The claim is trivial when $L$ is abelian. Then assume that condition (ii) of the statement holds and let $J$ be a restricted subalgebra of $L$. Let $x \in J$ and write $x=x_{s}+x_{n}$ with $x_{s} \in T$ and $x_{n} \in H$. Obviously, we have $\left[x_{s}, x_{n}\right]=0$ and then, as $x_{s}$ and $x_{n}$ are 2algebraic, so is $x$. Therefore $\operatorname{dim}\langle x\rangle_{2}<\infty$ and, as $F$ is perfect, we see that $x_{s}$ and $x_{n}$ are, respectively, the semisimple and the 2-nilpotent part of the JordanChevalley decomposition of $x$ (see [19], Chapter 2, Theorem 3.5); in particular we have $x_{n} \in\langle x\rangle_{2} \subseteq J$. Now, let $h$ be any non-central element of $H$ and suppose, if 
possible, that $h^{[2]} \in H^{[2]^{2}}$. Since $L$ is nilpotent of class 2 we have $H^{[2]} \subseteq Z(H)$, so that $h^{[2]}=z^{[2]}$ for some $z \in Z(H)$. It follows that

$$
(h+z)^{[2]}=0 ;
$$

thus $h+z \in \mathcal{N}(H) \subseteq Z(H)$ and then $h \in Z(H)$, a contradiction. Therefore $h^{[2]} \notin H^{[2]^{2}}=\left(H_{2}^{\prime}\right)^{[2]}$ and so, as $H_{2}^{\prime}$ is 2-nil and cyclic, it follows that $H_{2}^{\prime}=\left\langle h^{[2]}\right\rangle_{2}$. As a consequence, we have

$$
[x, L]=\left[x_{n}, H\right] \subseteq\left\langle x_{n}^{[2]}\right\rangle_{2} \subseteq J
$$

hence $J$ is an ideal of $L$.

Proving necessity is harder. This will be accomplished by splitting the proof into several steps.

Step 1. $\mathcal{N}(L) \subseteq Z(L)$. Let $v \in L$ such that $v^{[p]}=0$. Then $F v$ is a restricted Lie algebra of $L$ and thus, for every $w \in L$, we have $[v, w]=k v$ for some $k \in F$. Since $L$ is nilpotent (by Proposition 2.1), we have necessarily $k=0$; hence $v \in Z(L)$.

Step 2. If $F$ has odd characteristic, then $L$ is abelian. Assume $p>2$. From Proposition 2.1 it follows at once that every semisimple element is central in $L$. Since $L$ consists of $p$-algebraic elements and $F$ is perfect, for every $x \in L$ we can consider the Jordan-Chevalley decomposition $x=x_{s}+x_{n}$, where the summand $x_{s}$ is semisimple and $x_{n}$ is $p$-nilpotent. Therefore, in order to prove the claim it is enough to show that every $p$-nilpotent element $x$ of $L$ is contained in $Z(L)$. Let us proceed by induction on the exponent $n$ of $x$. For $n=1$ the claim follows from Step 1. Now, let $n>1$ and assume that every element of $L$ having exponent less than $n$ is central. Suppose ad absurdum that there is a $p$-nilpotent element $y$ of $L$ such that $[x, y] \neq 0$. By the inductive hypothesis, we can assume that $y$ has exponent $m \geq n$. Since $[x, y] \in\langle x\rangle_{p}$, we have

$$
[x, y]=\sum_{i=0}^{n-1} k_{i} x^{[p]^{i}}
$$

for suitable $k_{i} \in F$. Let $r:=\min \left\{i \mid k_{i} \neq 0\right\}$ and notice that $[x, y]$ is $p$-nilpotent of exponent $n-r$. Moreover, since $F$ is perfect, for every $i=r, \ldots, n-1$ there exists $\alpha_{i} \in F$ such that $\alpha_{i}^{p^{r}}=k_{i}$. Therefore, as [p] is $p$-semilinear by Proposition 2.1] we have

$$
[x, y]=\left(\sum_{i=r}^{n-1} \alpha_{i} x^{[p]^{i-r}}\right)^{[p]^{r}} .
$$

Furthermore, as $[x, y] \in\langle y\rangle_{p}$, by easy exponent arguments we have

$$
[x, y]=\left(\sum_{j=m-n+r}^{m-1} \beta_{j} y^{[p]^{j-r}}\right)^{[p]^{r}}
$$

for suitable $\beta_{m-n+r}, \ldots, \beta_{m-1} \in F$. As $[p]$ is $p$-semilinear, by (2.2) and (2.3) the element

$$
g:=\sum_{j=0}^{n-r-1}\left(\alpha_{j+r} x^{[p]^{j}}-\beta_{j+m-n+r} y^{[p]^{j+m-n}}\right)
$$


satisfies $g^{[p]^{r}}=0$; hence it has exponent less than $n$. From the inductive hypothesis it follows that $g$ is a central element of $L$ and so Proposition 2.1 yields

$$
0=[g, y]=\alpha_{r}[x, y] .
$$

As $\alpha_{r} \neq 0$, we conclude that $x$ and $y$ commute, a contradiction.

In the rest of the proof, assume $p=2$ and $L$ is not abelian.

Step 3. $L=T \oplus H$. Let $H$ be the set of all 2-nilpotent elements of $L$. For every $x \in H$ we clearly have $x^{[2]} \in H$ and, for every $y \in L$, from $[x, y] \in\langle x\rangle_{2}$ it follows that $[x, y]$ is 2-nilpotent, as well. Moreover, for every $\lambda, \mu \in F$ and $a, b \in H$, by Proposition 2.1 we see that also the element $(\lambda a+\mu b)^{[2]}=\lambda^{2} a^{[2]}+\mu^{2} b^{[2]}+\lambda \mu[a, b]$ is 2-nilpotent; thus $H$ is a restricted ideal of $L$. Now, let $T$ be the set consisting of all semisimple elements of $L$. It follows from Proposition 2.1 that $T \subseteq Z(L)$ and by [19] (Chapter 2, Proposition 3.3) $T$ is a torus, in particular a restricted ideal of $L$ with $T \cap H=\{0\}$. Since every $x \in L$ can be written as $x=x_{s}+x_{n}$ with $x_{s}$ semisimple and $x_{n}$ 2-nilpotent, we conclude that $L$ decomposes as $L=T \oplus H$.

Step 4. Elements of $H$ having different exponents commute. Proceed by induction on the exponent by following an argument similar to that used in the proof of Step 2.

Step 5. For all non-commuting elements $x$ and $y$ of $H$, one has $\langle[x, y]\rangle_{2}=\left\langle x^{[2]}\right\rangle_{2}=$ $\left\langle y^{[2]}\right\rangle_{2}$. By Step 4 the elements $x$ and $y$ have the same exponent, say $n$. Put $z:=[x, y]$. As in Step 2, we have

$$
z=\sum_{i=0}^{n-1} k_{i} x^{[2]^{i}}=\sum_{i=0}^{n-1} h_{i} y^{[2]^{i}},
$$

where $k_{0}, \ldots, k_{n-1}, h_{0}, \ldots, h_{n-1} \in F$. Since $L$ is nilpotent of class 2 , we clearly have $h_{0}=k_{0}=0$. In order to prove the claim it is enough to show that $k_{1} \neq 0$ and $h_{1} \neq 0$. We proceed by induction on $n$. The claim is trivial for $n \leq 2$. Assume then $n>2$ and let $r:=\min \left\{i \mid k_{i} \neq 0\right\}$ and $s:=\min \left\{i \mid h_{i} \neq 0\right\}$. Note that, by (2.4), we necessarily have $r=s$. If $r<n-1$, denote by $\tilde{x}$ and $\tilde{y}$ the images in $H /\left\langle x^{[2]^{n-1}}\right\rangle_{2}$ of $x$ and $y$, respectively. Then $\tilde{x}$ and $\tilde{y}$ have exponent $n-1$ and, by (2.4), the inductive hypothesis yields $k_{1} \neq 0$ and $h_{1} \neq 0$, as required. Now suppose by contradiction that $r=n-1$, so that

$$
z=k_{n-1} x^{[2]^{n-1}}=h_{n-1} y^{[2]^{n-1}} .
$$

Then, as $z^{[2]}=0$ and $n>2$, we have

$$
(\lambda x+\mu y)^{[2]^{n-1}}=0,
$$

where $\lambda^{2^{n-1}}=k_{n-1}$ and $\mu^{2^{n-1}}=h_{n-1}$. Consequently, the element

$$
\hat{x}:=\lambda x+\mu y
$$

has exponent less than $n$ and $[\hat{x}, y]=\lambda z \neq 0$, contradicting Step 4 .

Step 6. $H_{2}^{\prime}=\left\langle\bar{x}^{[2]}\right\rangle_{2}$ for any non-central element $\bar{x}$ of $H$. By Step 5 , for every $v \in H$ with $[v, \bar{x}] \neq 0$ we have

$$
\left\langle v^{[2]}\right\rangle_{2}=\langle[v, \bar{x}]\rangle_{2}=\left\langle\bar{x}^{[2]}\right\rangle_{2} .
$$

Now assume, if possible, that there are $a$ and $b$ in $H$ such that $[a, b] \notin\left\langle\bar{x}^{[2]}\right\rangle_{2}$. Then the previous discussion forces $[a, v]=[b, \bar{x}]=0$. 
Therefore, from $[\bar{x}+a, b] \neq 0$ and $[v, \bar{x}+a] \neq 0$ it follows that

$$
\begin{aligned}
\left\langle a^{[2]}\right\rangle_{2} & =\left\langle b^{[2]}\right\rangle_{2}=\langle[\bar{x}+a, b]\rangle_{2}=\left\langle(\bar{x}+a)^{[2]}\right\rangle_{2} \\
& =\langle[v, \bar{x}+a]\rangle_{2}=\langle[v, \bar{x}]\rangle_{2}=\left\langle\bar{x}^{[2]}\right\rangle_{2},
\end{aligned}
$$

so that

$$
[a, b] \in\left\langle a^{[2]}\right\rangle_{2}=\left\langle\bar{x}^{[2]}\right\rangle_{2},
$$

a contradiction. Hence we have

$$
H_{2}^{\prime}=\left\langle\bar{x}^{[2]}\right\rangle_{2}
$$

in particular, $H_{2}^{\prime}$ is cyclic.

Step 7. $H_{2}^{\prime}=H^{[2]}$. From what is proved in Step 6 , we have only to show that $w^{[2]}$ is in $H_{2}^{\prime}$ for every $w \in H$. By Step 5 , this is clear when $w \notin Z(H)$.

On the other hand, if $w \in Z(H)$, then, by Step 6, for every non-central element $\bar{x}$ of $H$ we have

$$
H_{2}^{\prime}=\left\langle\bar{x}^{[2]}\right\rangle_{2}=\left\langle(\bar{x}+w)^{[2]}\right\rangle_{2}=\left\langle\bar{x}^{[2]}+w^{[2]}\right\rangle_{2},
$$

so that $w^{[2]} \in H_{2}^{\prime}$, and the proof is complete.

At this stage, it is timely to understand for which perfect fields $F$ the class of restricted Lie algebras satisfying condition (ii) of Theorem 1.1 is actually not empty. Recall that $F$ is said to be 2-closed if it has no quadratic extension. Note that this is tantamount to saying that the polynomial $X^{2}+X+a$ is reducible in $F[X]$ for every $a \in F$. Thus, for example, every algebraically closed field is 2-closed, while for every positive integer $n$ the finite field $\mathbb{F}_{2^{n}}$ is not. We have the following result:

Proposition 2.2. Let $F$ be a perfect field of characteristic 2. Then the following conditions are equivalent:

(i) there exists a non-abelian restricted Lie algebra over $F$ with the property that all its restricted subalgebras are ideals;

(ii) $F$ is not 2-closed.

Proof. Assume first that $F$ is 2-closed and suppose, by contradiction, that there exists a non-abelian restricted Lie algebra $L$ over $F$ in the class $\mathcal{D}$. Then, by Theorem 1.1, there exist non-commuting 2-nilpotent elements $x$ and $y$ of $L$. By Step 5 in the proof of Theorem 1.1 we have

$$
\left\langle x^{[2]}\right\rangle_{2}=\left\langle y^{[2]}\right\rangle_{2}=\langle[x, y]\rangle_{2} .
$$

Denote by $\tilde{x}$ and $\tilde{y}$ the images in $\mathfrak{L}:=L /\left\langle x^{[2]^{2}}\right\rangle_{2}$ of $x$ and $y$, respectively. By (2.5) we have

$$
\begin{aligned}
{[\tilde{x}, \tilde{y}] } & =\alpha \tilde{x}^{[2]}, \\
\tilde{y}^{[2]} & =\beta \tilde{x}^{[2]},
\end{aligned}
$$

for some $\alpha, \beta \in F \backslash\{0\}$. Now, as $F$ is 2-closed, the polynomial $X^{2}+\alpha X+\beta$ has a root $\lambda$ in $F$. Consequently we have

$$
(\lambda \tilde{x}+\tilde{y})^{[2]}=\left(\lambda^{2}+\lambda \alpha+\beta\right) \tilde{x}^{[2]}=0
$$

so that from Theorem 1.1 it follows that $\lambda \tilde{x}+\tilde{y} \in Z(\mathfrak{L})$. Therefore we have $[\lambda \tilde{x}+\tilde{y}, \tilde{y}]=0$ and then, as $\lambda \neq 0$, this yields $[x, y] \in\left\langle x^{[2]^{2}}\right\rangle_{2}$, a contradiction to (2.5). 
Conversely, suppose that $F$ is not 2-closed and let $\zeta \in F$ such that the polynomial $X^{2}+X+\zeta$ is irreducible in $F[X]$. Consider the restricted Lie algebra $L$ over $F$ with a basis $\{a, b, c\}$ such that $[a, b]=c,[a, c]=[b, c]=0, a^{[2]}=c, b^{[2]}=\zeta c$, and $c^{[2]}=0$. Since $L$ is nilpotent, every subalgebra of $L$ of codimension 1 is an ideal. Moreover, for every $k_{1}, k_{2}, k_{3} \in F$, one has $\left(k_{1} a+k_{2} b+k_{3} c\right)^{[2]}=\left(k_{1}^{2}+k_{2}^{2} \zeta+k_{1} k_{2}\right) c$. From this, it follows at once that the only restricted 1-dimensional subalgebra of $L$ is $F c$. Consequently, every restricted subalgebra of $L$ is an ideal, and the claim follows.

Corollary 2.3. Let $(L,[p])$ be a restricted Lie algebra over an algebraically closed field of characteristic $p>0$. Then $L \in \mathcal{D}$ if and only if $L$ is abelian.

Remark 2.4. The class $\mathcal{D}$ is clearly closed under restricted subalgebras and homomorphic images but, in view of Theorem 1.1] and Corollary 2.3, it is not closed under direct sums or extensions of the ground field.

Finally, it should be noted that Theorem 1.1 fails for restricted Lie algebras over imperfect fields. In fact:

Example 2.5. Let $F$ be a field of characteristic $p>2$ containing an element $\alpha$ with no $p$-th root in $F$. Consider the restricted Lie algebra $L$ over $F$ with a basis $\{x, y, z\}$ such that $[x, y]=z,[x, z]=[y, z]=0, x^{[p]}=z, y^{[p]}=\alpha z$, and $z^{[p]}=0$. It is easy to check that any non-zero restricted subalgebra of $L$ contains $F z$. As a consequence, every restricted subalgebra of $L$ is an ideal, despite the fact that $L$ is not abelian.

\section{ACKNOWLEDGEMENT}

I am grateful to Professor Helmut Strade for many useful discussions.

\section{REFERENCES}

1. R.K. Amayo, Lie algebras in which every n-generator subalgebra is an n-step subideal, J. Algebra 31 (1974), 517-542. MR0347919 (50:419)

2. R.K. Amayo and I. Stewart, Infinite-dimensional Lie algebras, Noordhoff International Publishing, Leyden, 1974. MR0396708 (53:570)

3. R. Baer, Situation der Untergruppen und Struktur der Gruppe, S. B. Heidelberg. Akad. Wiss. 2 (1933), 12-17.

4. Yu. Bahturin and A. Olshanskii, Large restricted Lie algebras, J. Algebra 310 (2007), 413-427. MR2307801 (2008b:17027)

5. Y. Barnea and I.M. Isaacs, Lie algebras with few centralizer dimensions, J. Algebra 259 (2003), 284-299. MR 1953720(2004a:17005)

6. G. Benkart and I.M. Isaacs, Lie algebras with nilpotent centralizers, Can. J. Math. 31 (1979), 929-941. MR.546949 (81c:17022)

7. K. Bowman, D.A. Towers, and V.R. Varea, On flags and maximal chains of lower modular subalgebras of Lie algebras, J. Lie Theory 17 (2007), 605-616. MR2352003(2008h:17023)

8. R. Dedekind, Über Gruppen deren sämtliche Teiler Normalteiler sind, Math. Ann. 48 (1897), 548-561. MR1510943

9. M. Honda, On Lie algebras in which every subalgebra is a subideal, Hiroshima Math. J. 18 (1988), 655-660. MR991251 (90f:17029)

10. M. Lincoln and D.A. Towers, Frattini theory for restricted Lie algebras, Arch. Math. (Basel) 45 (1985), 451-457. MR815984 (88d:17007)

11. S. Liu, On algebras in which every subalgebra is an ideal, Acta. Math. Sinica 14 (1964), 532-537. MR0172899 (30:3115)

12. D.L. Outcalt, Power-associative algebras in which every subalgebra is an ideal, Pac. J. Math. 20 (1967), 481-485. MR0207784(34:7599) 
13. D.M. Riley, Restricted Lie algebras of maximal class, Bull. Austral. Math. Soc. 59 (1999), 217-223. MR1680807 (2000d:17023)

14. D.M. Riley and J.F. Semple, The coclass of a restricted Lie algebra, Bull. London Math. Soc. 26 (1994), 431-437. MR1308359 (96c:17014)

15. _ Completion of restricted Lie algebras, Israel J. Math. 86 (1994), 277-299. MR.1276139 (95d:17019)

16. S. Siciliano and Th. Weigel, On powerful and p-central restricted Lie algebras, Bull. Austral. Math. Soc. 75 (2007), 27-44. MR2309546 (2008b:17030)

17. I. Stewart, Infinite-dimensional Lie algebras in the spirit of infinite group theory, Compositio Mathematica 22 (1970), 313-331. MR0288159 (44:5357)

18. _ A note on 2-step subideals of Lie algebras, Compositio Mathematica 27 (1973), 273275. MR0335584 (49:365)

19. H. Strade and R. Farnsteiner, Modular Lie algebras and their representations, Marcel Dekker, New York, 1988. MR929682(89h:17021)

20. D.A. Towers, Elementary Lie algebras, J. London Math. Soc. (2) 7 (1973), 295-302. MR0376782 (51:12957)

21. (1980), 61-73. MR577906 (81f:17003)

22. — On complemented Lie algebras, J. London Math. Soc. (2) 22 (1980), 63-65. MR579809 (81h:17006)

23. On Lie algebras in which modular pairs of subalgebras are permutable, J. Algebra 68 (1981) 369-377. MR608540(82e:17010)

Dipartimento di Matematica "E. De Giorgi", Università del Salento, Via Provinciale LeCCe-Arnesano, 73100-Lecce, ItAly

E-mail address: salvatore.siciliano@unile.it 